\title{
BACTÉRIAS DO GÊNERO AEROMONAS NO FLUXOGRAMA DE BENEFICIAMENTO DO LEITE TIPO A E SEU COMPORTAMENTO FRENTE À AÇÃO DE ANTIMICROBIANOS
}

\author{
M.S. Carneiro; O.D .Rossi Junior
}

Universidade Estadual Paulista, Faculdade de Ciências Agrárias e Veterinárias, Departamento de Medicina Veterinária Preventiva e Reprodução Animal, Via de Acesso Prof. Paulo Donato Castellane, km 5, CEP 14884900, Jaboticabal, SP, Brasil.

\section{RESUMO}

\begin{abstract}
Objetivando verificar os possíveis pontos de contaminação e disseminação de bactérias do gênero Aeromonas, foram analisadas amostras de leite colhidas em diferentes pontos do fluxograma de beneficiamento em uma granja de leite tipo A. De 80 amostras analisadas, Aeromonas spp. foram isoladas em 37 (46,25\%) delas. Foram colhidas 20 amostras de cada ponto da linha de produção e revelaram-se positivas no isolamento, $90 \%$ (18/20) das amostras de leite cru, 30\% (6/ 20) das de leite da saída do pasteurizador, $40 \%(8 / 20)$ das de leite do tanque de abastecimento da máquina de empacotar e 25\% (5/20) das amostras do leite pronto para consumo. Dentre as espécies foram identificadas Aeromonas sobria em 40,54\% das amostras, Aeromonas hydrophila e Aeromonas caviae em 21,62\% delas, Aeromonas veronii em 10,81\% e Aeromonas schubertii em 2,7\%. Das cepas isoladas, 37 foram submetidas a testes de sensibilidade a antimicrobianos. Destas, $100 \%$ foram resistentes à ampicilina, 75,67\% à cefalotina, $64,86 \%$ à cefoxitina e $48,64 \%$ ao aztreonam. As drogas as quais as cepas mostraram-se menos resistentes foram, cloranfenicol, netilmicina, amicacina, gentamicina, tetraciclina e tobramicina. Os resultados obtidos demonstram que bactérias do gênero Aeromonas resistentes a vários antimicrobianos podem estar presentes no leite tipo A, o que faz com que este produto possa determinar risco à saúde da população consumidora, e que deve servir de alerta aos serviços de saúde pública.
\end{abstract}

PALAVRAS-CHAVE: Aeromonas spp., leite pasteurizado, antimicrobianos.

\section{ABSTRACT}

BACTERIA OF AEROMONAS GENUS IN THE TYPE “A” MILK PROCESSING FLOW AND THEIR BEHAVIOR IN REGARD TO ANTIMICROBIALS. Milk samples obtained from different points of the processing flow at a type " $\mathrm{A}$ " milk farm were analysed with the objective to verify possible points of Aeromonas genus bacteria contamination and dissemination. In 80 samples analysed, Aeromonas spp. were isolated in 37 (46.25\%) of them. From each point of the processing flow 20 samples were obtained. The results showed that $90 \%(18 / 20)$ of raw milk samples, 30\% $(6 / 20)$ of the pasteurizer-exit samples, $40 \%(8 / 20)$ of the packing-machine-tank samples and $25 \%$ $(5 / 20)$ of ready-to-drink milk samples were positive on isolation. The breakdown by species of the bacteria isolated was: Aeromonas sobria in $40.54 \%$ of the samples, Aeromonas hydrophila and Aeromonas caviae in $21.62 \%$, Aeromonas veronii in $10.81 \%$, and Aeromonas schubertii in $2.7 \%$. Of the isolated strains, 37 were submitted to antimicrobial susceptibility testing, which revealed that $100 \%$ were resistant to ampicillin, $75.64 \%$ to cephalotin, $64.86 \%$ to cefoxitin and $48.64 \%$ to aztreonam. The strains presented less resistance to chloramphenicol, netilmicin, amikacin, gentamicin, tetracycline and tobramycin. The results demonstrate that Aeromonas spp. bacteria resistant to several antimicrobials may be present in type " $\mathrm{A}$ " milk, indicating that this product might be a risk to consumer health, and this data should be an alert to public health services.

KEY WORDS: Aeromonas spp., pasteurized milk, antimicrobials.

\section{INTRODUÇÃO}

Nos anos recentes, surtos de doenças bacterianas de origem alimentar têm sido reportados por todo mundo, alguns causados por agentes chamados clássicos, os quais têm patogenia e epidemiologia bem conhecidas, como o Staphylococcus aureus, Clostridium perfringens e salmonelas, mas a grande maioria determinada por bactérias que não são comumente pesquisadas durante investigações de surtos. Dentre essas bactérias estão as aeromonas móveis (HANDFIELD et al., 1996). 
Aeromonas spp. são microrganismos de ocorrência amplamente difundida no meio ambiente (CUNLIFFE \& Adcock, 1989), sendo membros importantes da microbiota normal da água (PALumboetal.,1996), com relatos de isolamento a partir de água utilizada para os mais diversos fins (Rossi JúnIOR et al., 1996; GAVRIEL et al., 1998). A água, por sua vez, exerce papel de extrema importância como fonte de contaminação dos alimentos de origem animal (HÄNNINEN\&SIITONEN, 1995; Rossi JúNIOR et al., 2000).

O mecanismo de patogenicidade das aeromonas é considerado multifatorial, sendo sua ação classificada como de nível não intestinal e gastrentérica (Ko \& ChuAnG, 1995). Como fatores de virulência são atribuídos ao gênero Aeromonas a produção de enterotoxinas, citotoxinas, hemolisinas e proteases, além de grande capacidade de aderência e invasão em células da mucosa intestinal (SINGH \& SANYAL, 1992; KüHn et al., 1997; MARTINs et al., 2002).

Dentre os alimentos de origem animal Aeromonas spp. já foi isolado de carnes cruas, peixes, frutos do mar, aves, ovos, leite e derivados (ABEYTA JúNIOR et al., 1986; KIROv et al., 1993; PIN et al., 1994; Costa \& Rossi JÚNIOR, 2002; BulHÕES \& ROSSI JÚNIOR, 2002; Nocitietal., 1999; FreITAs et al., 1993). Particularmente, em relação ao leite bovino, a maioria dos trabalhos prende-se ao estudo do leitecru(KIROv etal.,1993;PRABHA etal.,1996; Melas et al., 1999) ou no final do fluxograma de produção, já pasteurizado e pronto para a comercialização (Freitas et al., 1993; ENEROTH et al., 2000; Craven \& Macauley, 1992).

Em vista do exposto, foi realizado o presente estudo com o objetivo de verificar a presença de bactérias do gênero Aeromonas em diferentes etapas do fluxograma de beneficiamento do leite tipo A e verificar o comportamento dos isolados frente à ação de antimicrobianos.

\section{MATERIAL E MÉTODOS}

O trabalho foi desenvolvido analisando amostras de leite ao longo do processo de beneficiamento em uma granja de leite tipo A localizada no interior do Estado de São Paulo. Foram analisadas amostras de leite cru da saída do pasteurizador, do tanque de abastecimento da máquina de envase e do leite empacotado pronto para a comercialização. No total foram analisadas 80 amostras, sendo 20 de cada um dos pontos estudados.

As amostras do leite do tanque de leite cru, do pasteurizador e do tanque de abastecimento da máquina de envase foram colhidas em frascos de vidro esterilizados, de $500 \mathrm{~mL}$ de capacidade, através de torneiras para a tomada de amostras existentes naqueles pontos. Os frascos, bem como o leite pasteuri- zado empacotado, foram acondicionados em caixas de material isotérmico com blocos de gelo e imediatamente transportados ao laboratório onde foram realizadas as análises.

O enriquecimento seletivo das amostras foi realizado após homogeneização manual, tomando-se 10 $\mathrm{mL}$ de cada uma delas e transferinda-os para frascos tipo Erlenmeyer contendo $100 \mathrm{~mL}$ de caldo tripticasesoja (TSB) acrescido de ampicilina na concentração de $30 \mathrm{mg} / \mathrm{L}$ (ABEYTA Junior et al., 1990). Após incubação a $28^{\circ} \mathrm{C}$ por $24 \mathrm{~h}$, as culturas foram semeadas em placas contendo ágar vermelho de fenol-amidoampicilina (PAlumboet al., 1985; MAJEed et al., 1990) e ágar dextrina-ampicilina (HAVELAAR \& VONK, 1988), as quais foram incubadas a $28^{\circ} \mathrm{C}$ por 24 h e examinadas quanto à presença de colônias grandes, de cor amarela, rodeadas por halo decorrente da hidrólise do amido ou da dextrina, respectivamente, características do gênero Aeromonas.

Colônias com características sugestivas foram semeadas em tubos com ágar tripticase-soja (TSA) inclinadoe, constatada a presença de culturas puras, elas foram semeadas em ágar tríplice-açúcar-ferro (TSI) e incubadas a $28^{\circ} \mathrm{C}$ por $24 \mathrm{~h}$ (SAAD et al.,1995). As culturas que apresentavam reação ácida tanto na base como no bisel, com ou sem formação de gás, foram submetidas às provas da motilidade, oxidase, catalase e resistência ao agente vibriostático $\mathrm{O} / 129$ (fosfato de 2,4-diamino-6,7-diisopropilpteridine), em esquema de identificação adotado por Popoff (1984) e Neves et al. (1990). A caracterização das espécies foi realizada seguind o o esquema dePopoff (1984) acrescido de outras provas recomendadas por BUCHANAN\& Palumbo (1985) e Abeyta Júnior et al. (1990). As culturas que deram reação distinta da classificação de POPOFF (1984) em apenas uma prova bioquímica foram designadas como atípicas, conforme adotado por MajeEd et al. (1990) e Kirov et al. (1993).

As espécies caracterizadas foram submetidas a testes de sensibilidade a antimicrobianos através da técnica de disco de difusão (BAUERet al., 1966; NCCLS, 2000). Utilizou-se polidiscos (Laborclin - LB6) com os princípios ativos: tetraciclina $(30 \mu \mathrm{g})$, cloranfenicol (30 $\mu \mathrm{g})$, netilmicina $(30 \mu \mathrm{g})$, aztreonam $(30 \mu \mathrm{g})$, gentamicina $(10 \mu \mathrm{g})$, amicacina $(30 \mu \mathrm{g})$, sulfazotrim $(25 \mu \mathrm{g})$, tobramicina $(10 \mu \mathrm{g})$, ampicilina $(10 \mu \mathrm{g})$, cefalotina (30 $\mu \mathrm{g})$, cefoxitina $(30 \mu \mathrm{g})$ e cefotaxima $(30 \mu \mathrm{g})$.

\section{RESULTADOS E DISCUSSÃO}

Os dados apresentados na Tabela 1 demonstram que, do total de 80 amostras analisadas, 37(46,25\%) revelaram-se positivas para a presença de bactérias do gênero Aeromonas, sendo que18(90\%) eram deleite cru, 6(30\%) doleite colhido na saída do pasteurizador, 
$8(40 \%)$ do leite do tanque de abastecimento da máquina de envase e 5 (25\%) amostras do leite empacotado pronto para o consumo. Nestas amostras foram identificadas as espécies A. hydrophila, A. sobria, $A$. caviae, A. veronii $A$. schubertii, além de vários isolados considerados como cepas atípicas.

A presença de Aeromonas sp. no leite cru tem sido demonstrada por vários autores, em diversas partes do mundo, com percentuais de amostras positivas bem inferiores aos $90 \%$ encontrados no presente estudo. SAAD (1991) obteve 38\% deamostras positivas colhidas em Assiut City, identificando a espécie A. hydrophila. PIN et al. (1994) na Espanha e URAZ \& ÇITAK (1998) na Turquia encontraram percentuais de $20 \%$ e $0,5 \%$, respectivamente, também com predominância dentre os isolados da espécie A. hydrophila. KIrOv etal. (1993), no entanto, encontraram positividade em $60 \%$ das amostras e identificaram as espécies $A$. sobria, A.hydrophila e A. caviae, além de cepas atípicas. PrabHa et al. (1996) identificaramas mesmas espéciesna Índia, e33,82\% de amostras positivas. Os autores citados são unânimes em afirmar que a origem desses microrganismos estaria relacionada a deficiências nos procedimentos de higiene na obtenção do leite. Com base nessas afirmações, eem vista dos resultados obtidos, pode-se afirmar que o percentual de $90 \%$ de amostras positivas verificado no presenteestudoé um forteindicativo defalhas no processo de obtenção.

Considerando-se ainda os dados da Tabela 1, referentes ao leite recém pasteurizado colhido na saída do pasteurizador e ao leite do tanque de abastecimento da máquina deempacotar, quenão deveriam encontrar-se contaminados, pois as Aeromonas spp são sensíveis ao calor e destruídas durante a pasteurização, verifica-se que foram encontradas 6 (30\%) amostras do primeiro produto e $8(40 \%)$ do segundo, positivas para o gênero Aeromonas. ENEROTH et al. (1998) analisando os pontos críticos de contaminação no fluxograma de laticínios na Suécia e Noruega, não verificaram contaminação do leite na saída do pasteurizador e tampouco no leite do tanque de abastecimento da máquina de envase.

Um fato que pode explicar a presença de aeromonas no leite pasteurizado é que as tubulações, o tanque de abastecimento da máquina de envase e a própria máquina de envase podem conter resíduos de água antes da passagem do leite. Sabe-se que estes resíduos podem abrigar um número considerável de microrganismos e os equipamentos, por estarem constantemente úmidos durante o dia de produção, se não forem apropriadamente limpos e secos no fim do dia podem permitir a formação de biofilmes, em decorrência da multiplicação dos microrganismos nas superfícies. IsONHOOD \& DRAKE (2002) afirmam que as aeromonas podem formar biofilmes eque estes contribuem no aumento da resistência aos tratamentos bactericidas normais.

No que diz respeito às amostras de leite pasteurizado prontas para consumo, o percentual de positividade encontrado, de $25 \%$ (5/20), foi superior ao verificado por KIROv et al. (1993), que obtiveram $4 \%$ de amostras positivas. Coincidentemente ao encontrado no presente estudo, a espécie que prevaleceu no leite pasteurizado foi a A. sobria, em cujas cepas os autores verificaram capacidade de produzir fatores de virulência como enterotoxinas, hemolisinas, citotoxinas e adesinas, caracterizando risco à saúde pública.

Os dados da Tabela 1 permitem verificar também que a Aeromonas caviae foi a espécie que determinou o segundo maior número de amostras positivas, 8 $(21,62 \%)$, isoladas em todos os tipos de amostras, exceto do leite recém pasteurizado colhido na saída do pasteurizador. O isolamento de A. caviae em leite tipo A pronto para consumo, que é considerado um leite de melhor qualidade, é outro fator que deve ser

Tabela 1 - Número de amostras positivas para cada uma das espécies de bactérias do gêneroAeromonas identificadas, em relação ao total analisado para cada um dos tipos de amostras colhidas em diferentes pontos do fluxograma de beneficiamento do leite tipo A.

\begin{tabular}{|c|c|c|c|c|c|c|c|}
\hline \multirow{2}{*}{$\begin{array}{l}\text { Tipo de } \\
\text { amostra }^{1}\end{array}$} & \multirow{2}{*}{$\begin{array}{c}\text { Total de amostras } \\
\text { positivas/analisadas }\end{array}$} & \multicolumn{6}{|c|}{ Espécies identificadas no de amostras (\%) } \\
\hline & & A. hydrophila & A. sobria & A. caviae & A. veronii & A. schubertii & atípicas \\
\hline LC & $18 / 20$ & $5(25)^{2}$ & $6(30)$ & $5(25)$ & $4(20)$ & $1(5)$ & $5(25)$ \\
\hline LSP & $6 / 20$ & $0(0)$ & $3(15)$ & $0(0)$ & $0(0)$ & $0(0)$ & $3(15)$ \\
\hline LTE & $8 / 20$ & $3(15)$ & $2(10)$ & $2(10)$ & $0(0)$ & $0(0)$ & $3(15)$ \\
\hline LE & $5 / 20$ & $0(0)$ & $4(20)$ & $1(5)$ & $0(0)$ & $0(0)$ & $0(0)$ \\
\hline Total & $37 / 80$ & $8(21,62)$ & $15(40,54)$ & $8(21,62)$ & $4(10,81)$ & $1(2,7)$ & $11(29,73)$ \\
\hline
\end{tabular}


Tabela 2 - Distribuição das cepas de Aeromonas hydrophila, A. sobria, A. caviae, A. veronii e atípicas, isoladas em diferentes pontos do fluxograma de beneficiamento do leite tipo A, resistentes a agentes antimicrobianos testados.

\begin{tabular}{lllllll}
\hline Antimicrobiano & \multicolumn{3}{c}{ № de cepas resistentes/testadas(\%) } & & $\begin{array}{l}\text { Total de cepas } \\
\text { resistentes }(\%)\end{array}$ \\
\cline { 2 - 5 } & A. hydrophila & A. sobria & A. caviae & A. veronii & atípicas & $3(8,11)^{1}$ \\
\hline Amicacina & $0 / 12(0 \%)$ & $2 / 8(25)$ & $0 / 8(0)$ & $0 / 4(0)$ & $1 / 5(20)$ & $37(100)$ \\
Ampicilina & $12 / 12(100)$ & $8 / 8(100)$ & $8 / 8(100)$ & $4 / 4(100)$ & $5 / 5(100)$ & $18(48,64)$ \\
Aztreonam & $7 / 12(58,33)$ & $4 / 8(50)$ & $4 / 8(50)$ & $0 / 4(0)$ & $3 / 5(60)$ & $28(75,67)$ \\
Cefalotina & $8 / 12(66,66)$ & $7 / 8(87,5)$ & $4 / 8(50)$ & $4 / 4(100)$ & $5 / 5(100)$ & $9(24,32)$ \\
Cefotaxima & $1 / 12(8,33)$ & $2 / 8(25)$ & $2 / 8(25)$ & $0 / 4(0)$ & $4 / 5(80)$ & $24(64,86)$ \\
Cefoxitina & $6 / 12(50)$ & $7 / 8(87)$ & $5 / 8(62,5)$ & $1 / 4(25)$ & $5 / 5(100)$ & $8(21,62)$ \\
Cloranfenicol & $1 / 12(8,33)$ & $2 / 8(25)$ & $1 / 8(12,5)$ & $1 / 4(25)$ & $3 / 5(60)$ & $9(24,32)$ \\
Gentamicina & $1 / 12(8,33)$ & $2 / 8(25)$ & $3 / 8(37,5)$ & $1 / 4(25)$ & $2 / 5(40)$ & $6(16,21)$ \\
Netilmicina & $0 / 12(0)$ & $2 / 8(25)$ & $1 / 8(12,5)$ & $0 / 4(0)$ & $3 / 5(60)$ & $15(40,54)$ \\
Sulfazotrim & $3 / 12(25)$ & $3 / 8(37,5)$ & $4 / 8(50)$ & $0 / 4(0)$ & $5 / 5(100)$ & $10(27,02)$ \\
Tetraciclina & $3 / 12(25)$ & $1 / 8(12,5)$ & $1 / 8(12,5)$ & $1 / 4(25)$ & $4 / 5(80)$ & $9(24,32)$ \\
Tobramicina & $2 / 12(16,66)$ & $2 / 8(25)$ & $0 / 8(0)$ & $1 / 4(25)$ & $4 / 5(80)$ & 9 \\
\hline
\end{tabular}

${ }^{1}$ Porcentagem em relação ao total de cepas testadas

levado em consideração pelos serviços de saúde pública, pois o leite é um alimento comum na dieta de muitas pessoas, incluindo grupos de alto risco, mais susceptíveis às infecções alimentares, ejá foi observado que aquela espécie, ainda que em menor grau, também produz fatores de virulência, conforme descrevem Cahill (1990) e HavelaAr et al. (1992). Segundo ReINA et al. (1991), a espécie A. caviae foi encontrada como principal enteropatógeno em casos de diarréia infantil em crianças submetidas a aleitamento artificial em Palma de Mallorca (Balares).

Analisando-se os dados da Tabela 2 verifica-se que o maior percentual de cepas resistentes, sem considerar a ampicilina, foi verificado frente a cefalotina $(75,67 \%)$, cefoxitina $(64,86 \%)$ e aztreonam $(48,64 \%)$. Para os demais princípios ativos testados os percentuais de cepas resistentes estiveram abaixo de $40 \%$. Ko et al. (1998) encontraram resultados semelhantes para cefalotina e cefoxitina em Tainan (Taiwan), ao analisarem cepas de A. hydrophilaisoladas de paciente com infecção. URRIZA et al. (2000), analisando cepas isoladas deágua de 2 rios europeus encontraram baixa resistência à gentamicina (1\%) e à tetraciclina $(14 \%)$ e alta resistência à gentamicina (93\%) o que difere do presente estudo. Nociтs et al. (1999) verificaram alto percentual de resistência a vários antimicrobianos entre cepas isoladas de carcaças de frango, concluindo pelo risco de infecções por microrganismos de alta resistência a partir do consumo daqueles produtos. Os autores citados são unânimes em afirmar que a emergência de resistência entre as Aeromonas spp. pode ser acelerada pelo mau uso dos antibióticos na terapêutica clínica, e também pela adição generalizada de subdoses em rações animais.
Considerando que as bactérias do gênero Aeromonas possuem capacidade de sobreviver e multiplicar em alimentos mantidos sob refrigeração, como o leite, e que muitas cepas são capazes de produzir toxinas, sua presença em leite pasteurizado, particularmente no tipo A, pode conferir a este produto um sério risco à saúde, principalmente, para o segmento da população considerado de alto risco, como crianças, idosos e pacientes imunodeprimidos.

\section{REFERÊNCIAS}

Abeyta Junior, C.; Kaysner, C.A.; WeKel, M.M.; Stott, R.F. Incidence of motile aeromonads from United States west coast shellfish growing estuaries. Journal of Food Protection, v.53, n.10, p.849-855, 1990.

AbeYta Junior, C.; KaYsner, C.A.; WeKel, M.M.;S Sullivan, J.J.; Stema, G.N. Recovery of Aeromonas hydrophila from oysters implicated in an outbreak of foodborneillness. Journal of Food Protection, v.49, p.643-646, 1986.

BAUER, A.W.;K IRBY,W.M.;S herris, J.C.; TURCK, M. Antibiotic susceptibility testing by a standardized single disk method. American Journal of Clinical Pathology, v.45, p.493-496, 1966.

Buchanan, R.L. \& Palumbo, S.A. Aeromonas hydrophilaand Aeromonas sobria as a potential food poisoning species: a review. Journal of Food Safety, v.17, p.1529,1985

BulHões, C.C.C. \& Rossi JúnIor, O.D. Ocorrência de bactérias do gênero Aeromonas em queijo de minas frescal artesanal. Arquivo Brasileiro de Medicina Veterinária e Zootecnia, v.54, n.3, p.320-324, 2002.

Cahill, M.M. Virulence factors in motile Aeromonas species. A review. Journal of Applied Bacteriology, v.69, p.1-16, 1990. 
Costa, F.N. \& Rossi Junior, O.D. Bactérias do gênero Aeromonas em abatedouro de frangos. Arquivo Brasileiro de Medicina Veterinária e Zootecnia, v.54,n.5, p.534538, 2002.

Craven, H.M. \& Macauley, B.J. Microorganisms in pasteurized milk after refrigerated storage. 1. Identification of types. Australian Journal of Dairy Technology, v.47, n.1, p.38-45, 1992.

Cunliffe, D.A. \& ADCock, P. Isolation of Aeromonas spp. from water by using anaerobic incubation. Applied and Environmental Microbiology, v.55, n.9, p.2138-2140, 1989.

Eneroth, A.; Ahrné, S.; Molin, G. Contamination of milk with gram-negative spoilage bacteria during filling of retail containers. International Journal of Food Microbiology, v.57, p.99-106, 2000.

Eneroth, A.; Christiansson, A.; Brendenhaug, J.; Molin, G. Critical contamination site in the production line of pasteurized milk, with reference to the psychrotrophic spoilage flora. International Dairy Journal, v.8, p.829834, 1998

Freitas, A.C.; Nunes, M.P.; Milhomem, A.M.; Ricciardi, J.D. Occurrence and characterization of Aeromonas species in pasteurized milk and white cheese in Rio de Janeiro, Brazil. Journal of Food Protection, v.50,n.1, p.6265, 1993.

Graviel, A.A.; Landre, J.P.B.; Lamb, J.A. Incidence of mesophilic Aeromonas within a public drinking water supply in north-east Scotland. Journal of Applied Microbiology, v.84, n.3, p.383-392, 1998.

HandField, M.; Simard,P.;C OUILlaRd,M.;Letarte, R.Aeromonas hydrophila isolated from food and drinking water: hemagglutination, hemolysis and cytotoxity for a humam humanintestinal cell line (HT-29). Appliedand Environmental Microbiology, v.62, n.9, p.3459-3461, 1996.

HÄNNINEN, M.L. \& SiItonen, A. Distribution of Aeromonas phenospecies and genospecies among strains isolated from water, foods or from human clinical samples. Epidemiolgy and Infection, v.115, p.39-50, 1995.

Havelaar, A.H.; Schets, F.M.; Van Silfhout, A.; Jansen, W.H.; Wieten, G. Typing of Aeromonas strains from patients with diarrhea and from drinking water. Journal of Applied Bacteriology, v.72, p.435-444, 1992.

HAVELAAR, A.H. \& VONK, M. The preparation of ampicillin dextrin agar for the enumeration of Aeromonas in water. Letters in Applied Microbiology, v.7, p.169-171, 1988.

Hühn, I.; Allestam, G.; Huys, G.; Janssen, P.; Kersters, K.; KROVACEK, K.; STEnSTRÖM, T.A. Diversity, persistence and virulence of Aeromonas strains isolated from drinking water distribution systems inSweden. Applied and Environmental Microbiology, v.63, n.7, p.2708-2715, 1997.

IsONHOOD, J. \& DrAKE, M.Aeromonas species in foods. Journal of Food Protection, v.65, n.3, p.575-582, 2002.

KIrov, S.M.; Huf, D.S.; HaYward, L.J. Milk as a potential source of Aeromonas gastrointestinal infection.Journal of Food Protection, v.56, n.4, p.306-312, 1993.

Ko, W.C. \& Chunng, Y.C. Aeromonas bacteremia: Review of 59 episodes. Clinical Infectious Diseases, v.20, p.12981304, 1995.
Ko,W.C.;Wu,H.M.;CHANG, T.C.; YAN, J.J.;Wu, J.J.Inducible B-lactamase resistance in A. hydrophila: therapeutic challenge for antimicrobial therapy. Journal of Clinical Microbiology, v.36, n.11, p.3188-3192, 1998.

MaJjed, K.N.; Egan, A.F.; Mac Rae, I.C. Production of exotoxins by Aeromonas sp. at $5^{\circ} \mathrm{C}$. Journal of Applied Bacteriology, v.69, p.332-337, 1990.

Martins, L.M.; MARQuez, R.F.; YANO, T. Incidence of toxic Aeromonas isolated from food in human infection. FEMSImmunologyand Medical Microbiology, v.32, n.237, p.237-242, 2002.

MELAS, D.S.;P APAGEORGIOU, D.M.; MANTIS, A.L.Enumeration and confirmation of Aeromonas hydrophila, Aeromonas caviae and Aeromonas sobria isolated from raw milk and other milk products in Northern Greece. Journal of Food Protection, v.62, n.5, p.463-466, 1999.

National Committee for Clinical Laboratory Standards NCCLS. Methods for dilution antimicrobial susceptibility tests for bacteria that grow aerobically; Approved Standard-Fifth Edition. NCCLSdocument M7-45, 2000.

Neves, M.S.; NunEs, M.P.; Ricciardi, I.D. Incidence of motile Aeromonas species in aquatic environments of Rio de Janeiro, Brazil. Journal of Food Protection, v.53, n.1, p.78-80, 1990

Nociti, D.L.P.; Ross Junior, O.D.; AmaraL, L.A. Bactérias do gênero Aeromonas em carcaças e cortes comerciais de frangos comercializados em Jaboticabal, Estado de São Paulo e comportamento das cepas frente a ação de antimicrobianos. Revista Brasileira de Ciência Veterinária, v.6, n.2, p.69-73, 1999.

Palumbo, S.A.; Call, J.; Huynh, B.; Fanelli, J. Survival an growth potential of Aeromonas hydrophila in reconditioned pork-processing-plant water. Journal of Food Protection, v.59, n.8, p.881-885, 1996.

Palumbo, S.A.; Maxino, F.; Willians, A.C.; Buchanan, R.L.; Thayer, D.W. Starch-Ampicilin Agar for the quantitative detection of Aeromonas hydrophila.Applied and Environmental Microbiology, v.50, n.4, p.1027-1030, 1985.

Pin, C.; Marin, M.L.; Garcia, M.L.; Tormo, J.; Selgas, M.D.; CASA, C. Incidence of motile Aeromonas spp in foods. Microbiologia SEM, v.10, p.257-262, 1994.

Popoff, M. Genus III. Aeromonas Kluyver and Van Neil. In: Noel R. Drieg (ed.), Bergey's manual of systematic bacteriology. Baltimore: Williams and Wilkins, 1984. v.1, p.545-548.

Prabha, R.; KRIShNA, R.; SHANKAR, P.A. Identification of gram-negative rod shape psychrotrophic bacteria of dairy origin. Indian Journal of Dairy Science, v.49, n.8, p.517-524, 1996.

Reina, J.; Lompart, I.; Gómez, J.; Borrell, N., Serra, A. Aeromonas caviae: principal especie enteropatogena del grupo de las Aeromonas mesófilas durante el período de lactancia artificial en la plobacion de Palma de Mallorca (Balares). Revista Espanhola de Pediatria, v.47, n.2, p.146-150, 1991.

Rossi Júnior, O.D.; Amaral, L.A.; NAder Filho, A. Bactérias do gênero Aeromonas em água de matadouro bovino. Arquivo Brasileiro de Medicina Veterinária e Zootecnia, v.52, n.5, p.549-553, 2000. 
Rossi Júnior, O.D.; SAntos, I.F.; Amaral, L.A.; B ARbosa, A.M. Aeromonas species research in samples of water and beef obtained at industrial level. Revista Brasileira de Ciência Veterinária, v.3, n.3, p.75-78, 1996.

SAAD, N. Occurrence of Aeromonas hydrophila en raw milk. Assuit Veterinary Medical Journal, v.25, n.50, p.98-102, 1991.

SAAD, S.M.I.; I ARIA, S.T.;F URLANETTO, S.M.P. MotileAeromonas spp in retail vegetables from São Paulo, Brazil.Revista de Microbiologia, v.26, n.1, p.22-27, 1995.

SingH, C.V. \& SANYAL, S.C. Enterotoxicity of clinical and environmental isolates of Aeromonas spp. Journal of Medical Microbiology, v.35, p.269-272, 1992.
URAZ, G.\&Ç ITAK, S. The isolation of Pseudomonas and other gram negative psychrotrophic bacteria in raw milk. Journal of Basic Microbiology, v.38, n.2, p.129-134, 1998.

Urriza, M.G.; Pineau, L.; CapdePuy, M.; Roques, C.; Caumatte, P.;Quentin, C. Antimicrobial resistance of mesophilic Aeromonas spp isolated from two European rivers. Journal of Antimicrobial Chemotherapy, v.46, p.297-301, 2000.

Recebido em $24 / 5 / 06$

Aceito em 23/8/06 